\title{
A VANISHING THEOREM FOR SEIBERG-WITTEN INVARIANTS
}

\author{
Shuguang WANG
}

\begin{abstract}
A вStRACт. It is shown that the quotients of Kähler surfaces under free
\end{abstract} anti-holomorphic involutions have vanishing Seiberg-Witten invariants.

Various vanishing theorems have played important roles in gauge theory. The first among them, due to S. K. Donaldson [2], states that the Donaldson invariants vanish for any smooth closed oriented 4-manifold $X$ which decomposes to a connected sum $X_{1} \# X_{2}$ with $b_{2}^{+}\left(X_{1}\right)>0, b_{2}^{+}\left(X_{2}\right)>0$. (Here $b_{2}^{+}\left(X_{i}\right)$ is the dimension of a maximal subspace of $H_{2}\left(X_{i}, \mathbf{Z}\right)$ on which the intersection pairing is positive definite.) E. Witten [12] has shown more recently that such a connected-sum manifold has also vanishing SeibergWitten invariants. Even though its proof is simple, Witten's vanishing theorem is equally useful as Donaldson's vanishing theorem; for example, it implies that any symplectic 4-manifold cannot be decomposed into the above connected sum by combining with Taubes' theorem in [9].

In this note we show that Seiberg-Witten invariants vanish for another class of 4-manifolds. These manifolds are obtained in connection with real algebraic geometry, and the construction was originally proposed in Donaldson [3]. See Remark 4 below. To state our theorem, recall that a map $\sigma$ between two almost complex manifolds is called anti-holomorphic if $\sigma_{*} J_{1}=-J_{2} \sigma_{*}$ on the tangent bundles, where $J_{i}$ are the almost complex structures of the manifolds. In the following, $K$ denotes the canonical bundle of an almost complex manifold (underlying a Kähler manifold).

Theorem 1. Let $\widetilde{X}$ be a Kähler surface with $K_{\tilde{X}}^{2}>0$ and $b_{2}^{+}(\widetilde{X})>3$. Suppose that $\sigma: \tilde{X} \rightarrow \widetilde{X}$ is an anti-holomorphic involution without fixed

1991 Mathematics Subject Classification. Primary 57R55, 57R57, 57N13; Secondary $14 \mathrm{P} 25$.

Received April 14, 1995.

Work supported by the Research Board grant of University of Missouri. 
points. Then the quotient manifold $X=\widetilde{X} / \sigma$ has vanishing Seiberg-Witten invariants.

In view of Witten's vanishing theorem, it is natural to examine whether the quotient $X$ can be decomposed into a connected sum:

Proposition 2. If $\widetilde{X}$ is a simply-connected Kähler surface or more generally symplectic 4-manifold with $b_{2}^{+}(\widetilde{X})>1$ and $\sigma: \widetilde{X} \rightarrow \widetilde{X}$ is a free involution, then the quotient manifold $X=\widetilde{X} / \sigma$ is not diffeomorphic to any connected sum $X_{1} \# X_{2}$ with both $b_{2}^{+}\left(X_{i}\right)>0$.

Proof. Suppose on the contrary, that there is such a decomposition $X=$ $X_{1} \# X_{2}$. Since $\pi_{1}(X)=\mathbf{Z}_{2}$, we can assume $\pi_{1}\left(X_{1}\right)=\mathbf{Z}_{2}$ and $\pi_{1}\left(X_{2}\right)=1$. A universal (double) cover $\widetilde{X}_{1}$ of $X_{1}$ yields a universal cover $\widetilde{X}_{1} \# X_{2} \# X_{2}$ of $X$, which should then be diffeomorphic to $\widetilde{X}$. This is however impossible as $\widetilde{X}$, being symplectic, cannot be decomposed into such a connected sum with $b_{2}^{+}\left(\widetilde{X}_{1} \# X_{2}\right)>0$ and $b_{2}^{+}\left(X_{2}\right)>0[9]$. (This kind of covering trick was initially used in [7] in a different context.)

Proposition 2 therefore indicates that at least in the case where $\widetilde{X}$ is simply-connected, the situation in Theorem 1 is not covered by Witten's vanishing theorem. To the author's knowledge, the quotient manifold $X$ given here, with $b_{1}(X)+b_{2}^{+}(X)=b_{2}^{+}(X)$ odd, is the first kind of example which is known to satisfy the conclusions in Theorem 1 and Proposition 2.

Before proving Theorem 1, we recall briefly the definition of SeibergWitten invariants from [8] and [12], for example. Let $Y$ be a smooth oriented Riemannian 4-manifold. In this case, a $\operatorname{spin}^{c}$ structure $\lambda$ on $Y$ consists of a pair of $U(2)$ bundles $W^{ \pm}$over $Y$, whose sum $W^{+} \oplus W^{-}$is the usual spinor bundle. Let $L$ denote the determinant (complex line) bundle of $W^{+}$. The perturbed Seiberg-Witten equations are the following pair of equations for a unitary connection $A$ on $L$ and a section $\phi$ of $W^{+}$:

$$
\begin{aligned}
D_{A} \phi & =0 \\
\rho\left(F_{A}^{+}+i \delta\right) & =i \theta(\phi, \phi),
\end{aligned}
$$

Here $D_{A}: \Gamma\left(W^{+}\right) \rightarrow \Gamma\left(W^{-}\right)$is the Dirac operator, $F_{A}^{+}$is the self-dual part of the curvature of $A, \rho$ is the Clifford multiplication, and $\theta$ is a pairing defined by matrix multiplication. (See $[8]$ for details.) For a generic perturbation $\delta \in \Omega^{+}(X)$, the moduli space $M_{\lambda, \delta}$ of irreducible solutions $(A, \phi)$ is either empty or a smooth manifold of dimension

$$
d_{\lambda}=\frac{1}{4}\left[c_{1}(L)^{2}-\left(2 e_{Y}+3 s_{Y}\right)\right]
$$


where $e_{Y}, s_{Y}$ are respectively the Euler characteristic and signature of $Y$. (The pair $(A, \phi)$ is called irreducible if $\phi \neq 0$.) The moduli space $M_{\lambda, \delta}$ will be compact if the metric on $Y$ is chosen so that $(*)$ admit no reducible solutions, which can be achieved in a path-connected subset of metrics if $b_{2}^{+}(Y)>1$. In this situation and if $d_{\lambda} \geq 0$ also, the Seiberg-Witten invariant $\mathrm{SW}(Y, \lambda)$ of $Y$ with respect to $\lambda$ is then defined to be the integral of the maximal power of the Chern class of the circle bundle $M_{\lambda, \delta}^{0} \rightarrow M_{\lambda, \delta}$, where $M_{\lambda, \delta}^{0}$ is the framed moduli space. (The integral makes sense only if $b_{1}(Y)+b_{2}^{+}(Y)$ is odd; if $b_{1}(Y)+b_{2}^{+}(Y)$ is even, the Seiberg-Witten invariant is defined to be zero.)

The full details of the definition of Seiberg-Witten invariants will not be needed in this paper. In fact, for the purpose of proving Theorem 1, it is enough to note that the perturbation $\delta$ will be generic and the SeibergWitten invariant will be zero if there are no reducible and irreducible solutions to $(*)$. The issue of reducible solutions is dealt with in the following simple observation.

Lemma 3. Set $\delta=0$ in (*). If the smooth 4-manifold $Y$ satisfies $2 e_{Y}+$ $3 s_{Y}>0$, then there is no reducible solution to (*) for any metric and any spin $^{c}$ structure $\lambda$ on $Y$ with $d_{\lambda} \geq 0$.

Proof. Since $d_{\lambda}=\frac{1}{4}\left[c_{1}(L)^{2}-\left(2 e_{Y}+3 s_{Y}\right)\right]$, its nonnegativeness yields $c_{1}(L)^{2}>0$. If there is a reducible Seiberg-Witten solution for some metric, that is, a connection $A$ on $L$ with $\frac{i}{2 \pi} F_{A}$ being anti-self dual, then $c_{1}(L)^{2}=\int_{Y}\left(\frac{i}{2 \pi} F_{A}\right)^{2} \leq 0$. This is a contradiction.

It is interesting to observe that the situation in Lemma 3 is different from the Donaldson theory. There the inequality $c_{1}(L)^{2} \leq 0$ (from a reducible anti-self dual connection on an $\mathrm{SU}(2)$ bundle $E$ ) does not contradict the nonnegativity of the (virtual) dimension $d_{E}=8 c_{2}(E)-3\left(e_{Y}+s_{Y}\right) / 2$ of the moduli space of ASD connections, as $c_{2}(E)=-c_{1}(L)^{2} \geq 0$. Thus a condition such as $e_{Y}+s_{Y}>0$ is not helpful to rule out the existence of reducible ASD connections.

Proof of Theorem 1. Let $h$ be the Kähler metric on $\widetilde{X}$ and $\omega$ the associated Kähler form. As $\sigma$ is anti-holomorphic, one sees easily that $\widetilde{g}=h+\sigma^{*} h$ is an equivariant Kähler metric on $\widetilde{X}$ with Kähler form $\widetilde{\omega}=\omega-\sigma^{*} \omega$. Through the double cover $p: \widetilde{X} \rightarrow X, \widetilde{g}$ pushes down to a metric $g$ on $X$. Both $\widetilde{g}$ and $g$ will be fixed for the rest of the proof. The standard Euler characteristic and signature formulae applied to $p$ also yield $b_{2}^{+}(X)=\frac{1}{2}\left[b_{2}^{+}(\widetilde{X})-1\right]>1$.

Consider an arbitrary $\operatorname{spin}^{c}$ structure $\lambda$ on $X$. It pulls back to a $\operatorname{spin}^{c}$ structure $\tilde{\lambda}$ on $\widetilde{X}$ through the double covering $p$. Without much difficulty one verifies that the associated bundles $W^{ \pm}$and $L$ of $\lambda$ pull back to the 
corresponding associated bundles $\widetilde{W}^{ \pm}$and $\widetilde{L}$ of $\widetilde{\lambda}$. Assume the dimension $d_{\lambda} \geq 0$ from now on.

Suppose there is a solution pair $(A, \phi)$ to the Seiberg-Witten equations:

$$
\begin{aligned}
D_{A} \phi & =0 \\
\rho\left(F_{A}^{+}\right) & =i \theta(\phi, \phi),
\end{aligned}
$$

where $A$ and $\phi$ are respectively a connection on $L$ and a section on $W^{+}$. Then the pull-back $(\widetilde{A}, \widetilde{\phi})$ is a solution pair to the Seiberg-Witten equations on $\widetilde{X}$ with $\operatorname{spin}^{c}$ structure $\tilde{\lambda}$ and Kähler metric $\widetilde{g}$. Since $2 e_{X}+3 s_{X}=\left(2 e_{X}+\right.$ $\left.3 s_{X}\right) / 2=K_{X}^{2} / 2>0$, there are no reducible Seiberg-Witten solutions for $\lambda$ from Lemma 3 ; thus $(A, \phi)$ and hence $(\widetilde{A}, \widetilde{\phi})$ are both irreducible. It follows easily from the irreducibility of $(\widetilde{A}, \widetilde{\phi})$ that $\widetilde{\omega} \cdot \widetilde{L} \neq 0$ (see [12] for example). This is however not possible; $\sigma^{*} \widetilde{L}=\widetilde{L}$ and $\sigma^{*} \widetilde{\omega}=-\widetilde{\omega}$ have already forced $\widetilde{\omega} \cdot \widetilde{L}=0$. (Note that $\sigma$ preserves the orientation of $\widetilde{X}$.)

Thus the argument above shows that there is no reducible or irreducible solution to $(* *)$. As noted before Lemma 3, this means that in the perturbed Seiberg-Witten equations of $(* *)$, the perturbation $\delta=0$ is generic and the Seiberg-Witten invariant of $X$ with respect to $\lambda$ is zero. Since $\lambda$ is an arbitrary $\operatorname{spin}^{c}$ structure, the Seiberg-Witten invariants of $X$ all vanish.

Note that all minimal complex surfaces of general type satisfy the condition $K_{X}^{2}>0$ in Theorem 1 [1; page 208]. (Moreover a necessary condition for $K_{X}^{2}>0$ is that $\widetilde{X}$ be projective by using Grauert's ampleness criterion [1; Page 127].)

Therefore the conditions in both Theorem 1 and Proposition 2 are satisfied by all simply-connected minimal complex surfaces of general type with $b_{2}^{+}>3$, and these include lots of examples. For one set of examples, one can take $\widetilde{X}$ to be algebraic surfaces in $\mathbf{C P}^{n}$ defined by real polynomials and $\sigma$ to be the complex conjugation. These include hypersurfaces $\sum_{j=1}^{4} x_{j}^{2 n}=0$ in $\mathbf{C P}^{3}$, where $n>2$. For another set of examples, consider a Kähler surface $Y$ with anti-holomorphic involution $\tau$. Suppose that there exits a complex curve $C \subset Y$ such that $2 \mid[C] \in H_{2}(Y, \mathbf{Z})$, and that $C$ is invariant under $\tau$, disjoint from Fix $\tau$. Then $\tau$ lifts to two anti-holomorphic involutions on the double cover $\tilde{X}$ of $Y$ branched over $C$, and one of the lifting involutions has no fixed point. As a special case, one can take $Y=\mathbf{C P}^{2}$, $C=\left\{\sum_{j=1}^{3} x_{j}^{2 n}=0\right\}(n>3)$ and $\tau$ to be the complex conjugation on $\mathbf{C P}^{2}$. 
Remark 4.

(i) Since the Seiberg-Witten invariants vanish, it follows from [9] that the quotient $X$ in Theorem 1 does not admit any symplectic structure.

Moreover it is possible to give examples of $\widetilde{X}$ (e.g., degree $4 m+2$ hypersurfaces in $\mathbf{C P}^{3}$ ), from which $b_{2}^{+}(X), b_{2}^{-}(X)$ are both even. Thus $X$ does not even have an almost complex structure with either of its orientations in this case.

(ii) Fix a complex surface $\widetilde{X}$ but vary free anti-holomorphic involutions $\sigma$. It is an open problem whether the quotients $X$ are diffeomorphic to each other. (It is not difficult to see that the quotients are homeomorphic to each other for most cases using [6].) Perhaps the d-complex structures introduced in [11] are a useful tool.

(iii) It is also interesting to investigate the quotient $X$ when $\sigma$ is not free. By using the generalized adjunction inequality [4], one can show easily that the Seiberg-Witten invariants of $X$ vanish again for many cases. (Furthermore, combining with the rational blowdown formula [5] as well as a surgery formula [10], one can recover the vanishing result in Theorem 1 for hypersurfaces in $\mathbf{C P}^{3}$.) It remains unknown [3] whether there exits such an $X$ which cannot be decomposed into a sum $X_{1} \# X_{2}$ with both $b_{2}^{+}\left(X_{i}\right)>0$ (other than a couple of trivial cases with $b_{2}^{+}(X)=0$ ).

\section{Acknowledgements}

The author wishes to thank Ron Fintushel for pointing out the incompleteness of a version of the paper, and Ron Stern for valuable encouragement. In addition, he thanks Jan Segert for sharing the enthusiasm about the new theory.

\section{References}

1. W. Barth, C. Peters and A. Van de Ven, Compact complex surfaces, Springer-Verlag, Berlin-Heidelberg, 1984.

2. S. Donaldson, Polynomial invariants for smooth 4-manifolds, Topology 29 (1990), 257-315.

3. _ Y Yang-Mills invariants of 4-manifolds, in Geometry of low-dimensional manifolds (S. K. Donaldson and C. B. Thomas Eds, eds.), vol. 1, Cambridge University Press, 1990, pp. 5-41.

4. R. Fintushel, P. Kronheimer, T. Mrowka, R. Stern and C. Taubes, in preparation.

5. R. Fintushel and R. Stern, in preparation.

6. I. Hambleton and M. Kreck, Smooth structures on algebraic surfaces with cyclic fundamental group, Invt. Math. 91 (1988), 53-59. 
7. D. Kotschick, On connected sum decompositions of algebraic surfaces and their fundamental groups, Intern. Math. Research Notices (1993), 179-182.

8. P. Kronheimer and T. Mrowka, The genus of embedded surfaces in the projective plane, Math. Research Letters 1 (1994), 797-808.

9. C. Taubes, The Seiberg-Witten invariants and symplectic forms, Math. Research Letters 1 (1994), 809-822.

10. S. Wang, On quotients of real algebraic surfaces in $\mathbf{C P}^{3}$, Topology and Its Applications (to appear).

11. _ A Narasimhan-Seshadri-Donaldson correspondence on non-orientable surfaces, Forum Mathematicae (to appear).

12. E. Witten, Monopoles and 4-manifolds, Math. Research Letters 1 (1994), 769-796.

M athematics Department, University of M issouri, Columbia，M O 65211

E-mail address: sw@wang.cs.missouri.edu 